\title{
Clinical audit of management of smear positive adult pulmonary tuberculosis in PR1 tuberculosis clinic, KK Bandar Miri
}

\author{
Rubini a/p Cumarasamy, Fatimah Shahidah Binti Dato' Azmi, Norafini Binti Salamon \\ Cumarasamy R, Azmi FS, Salamon N. Clinical audit of management of smear positive adult pulmonary tuberculosis in PR1 tuberculosis clinic, \\ KK Bandar Miri. Malays Fam Physician. 2021;16(2);50-57. https://doi.org/10.51866/oa1215
}

\section{Keywords:}

Audit, pulmonary TB,

management, smear positive,

KK Bandar Miri

\section{Authors:}

Rubini a/p Cumarasamy
(Corresponding author)
MBBS (UnisZa)
Klinik Kesihatan Bandar Miri
Ministry of health, Malaysia
Email: rubzcumar@gmail.com

Fatimah Shahidah Binti Dato'

Azmi

$M D(C S M U)$

Klinik Kesihatan Bandar Miri

Ministry of health, Malaysia

Norafini Binti Salamon

MD (UNIMAS), MMed FamMed (UKM)

Klinik Kesihatan Bandar Miri

Ministry of health, Malaysia

\begin{abstract}
Introduction: Malaysian tuberculosis (TB) clinical practice guideline (CPG) standardises the management of TB to improve the quality of care for TB patients. Not all primary care providers adhere to this guideline, however. This audit aims to improve the management of smear positive adult pulmonary TB by identifying areas of concern and developing action plans.

Methods: Data for the audit were gathered from August 2018 to August 2019 from medical records of diagnosed smear positive pulmonary TB patients. Patients were included based on inclusion and exclusion criteria.

Results: Forty-eight smear positive pulmonary TB patients were recruited. The majority of patients were male (54.2\%) and Malay (33.2\%). TB symptoms were assessed for $29.2 \%$ of patients at two weeks and then in $81.3 \%$ at one month, $97.9 \%$ at two months, $16.7 \%$ at three months, $95.8 \%$ at four months, $22.9 \%$ at five months and $95.8 \%$ at six months. Medication side effects were assessed at two weeks for only $2.1 \%$ of patients, then for $8.3 \%$ of patients at one month, $2.1 \%$ at two months and $0 \%$ at subsequent months. At diagnosis, $25 \%$ and $4.2 \%$ of patients had their visual acuity and colour vision, respectively, assessed. Only $6.3 \%$ of patients were referred to pre-pregnancy counselling. Weights were recorded for $87.5 \%$ of patients at diagnosis, then for $27.1 \%$ at two weeks, $81.3 \%$ at one month, $91.7 \%$ at two months, $77.1 \%$ at three months, $97.9 \%$ at four months, $79.2 \%$ at five months and $93.8 \%$ at six months. Baseline investigations and Mycobacterium tuberculosis culture and sensitivity were conducted for all patients, but only $81.3 \%$ of patients were traced. No patients were referred to pharmacy for medication counselling.

Conclusion: This audit shows that there is a difference between current practice and the national guideline. There are some downfalls in management and proper documentation, so interventions should be carried out to improve those aspects.
\end{abstract}

\section{Introduction}

Tuberculosis (TB) remains one of the world's deadliest communicable diseases. ${ }^{1}$ The number of cases in Malaysia continues to rise unabated, leading to high rates of morbidity and mortality. ${ }^{1}$ Sabah was noted to have the highest prevalence of TB cases, followed by Selangor and Sarawak. ${ }^{2}$ The number of new TB cases in the country increased from 15,000 in 2005 to 19,251 in 2011 , with $72 \%$ of new pulmonary tuberculosis (PTB) cases being smear positive. ${ }^{3}$

The management of TB must be standardised to improve patient outcomes, assist in monitoring, evaluate adherence to achieve a cure, prevent mortality and morbidity, reduce transmission of tuberculosis and prevent emergence of multidrug-resistant TB. ${ }^{2}$ Hence, the Malaysian TB clinical practice guideline (CPG) standardises the management of $\mathrm{TB}$ at all levels of care in Malaysia to improve quality of care for TB patients. ${ }^{2}$ Primary care providers, however, may not adhere to the $\mathrm{CPG}^{2}$

Smear positive PTB is diagnosed with a suggestive chest radiograph and one sputum smear positive for acid-fast bacilli resembling Mycobacterium tuberculosis. ${ }^{3}$

A clinical audit evaluates if a certain aspect of health care is attaining an appropriate standard. This allows healthcare providers and patients to assess the quality of service and allow room for improvements. ${ }^{4}$ Audits help organisations to continuously work towards improving quality of care by showing pitfalls, implementing improvements and re-auditing to see if improvements have occurred. ${ }^{4}$ 
Clinical record keeping, regardless of the form of the records (e.g. electronic or paper), is an integral component of good professional practice and delivery of quality healthcare. ${ }^{5}$ These records are also valuable for auditing the quality of services provided. ${ }^{5}$ Thus, the aim of this audit was to assess the quality of care based on obtaining proper history, proper documentation in monitoring of side effects and correct management towards smear positive PTB patients in Klinik Kesihatan Bandar Miri, Sarawak, as required in our National Standard Clinical Practice Guideline. ${ }^{4}$

Clear, prompt and accurate record keeping of the TB register, treatment card and any patientheld cards is necessary to monitor progression of each patient and their treatment. ${ }^{6}$

\section{Objectives}

The objectives of this audit are:

- To improve management of adults with smear positive PTB at the Pusat Rawatan 1 (PR1) TB clinic at KK Bandar Miri.

- To identify areas of concern and develop action plans to resolve them.

\section{Methodology \\ Settings}

KK Bandar Miri serves an average population of 20,000 patients. Service in the PR1 TB clinic is provided by one medical officer, one medical assistant, one staff nurse and one community nurse. This clinic is fully equipped with laboratory facilities, radiology department, pharmacy and PR1 for treatment and contact tracing.

\section{Process of Care}

This clinical audit was conducted in PR1 at KK Bandar Miri. The inclusion criteria included adult smear positive PTB patients aged 18 and above. The exclusion criteria were smear negative PTB and extrapulmonary TB.

Forty-eight adult smear PTB positive medical records were recruited from a total of 138 patients diagnosed with smear positive PTB, smear negative PTB and extrapulmonary TB within a one-year time frame, August 2018 to August 2019. The required data were gathered retrospectively from the medical records of diagnosed smear positive PTB patients kept at PR1, KK Bandar Miri.

Only smear positive PTB patients were selected for this audit to avoid false positive cases such as malignancy or nontuberculous Mycobacterium infections. Extrapulmonary TB was not selected, as the duration of treatment is more than six months, which was outside the six-month duration of our audit.

In a clinical audit, a small sample size is usually sufficient, roughly $20-50$ cases. For this audit, we selected patients over the course of a one-year period. All 48 smear positive PTB patients were selected.

Firstly, the indicators of this audit were set, which consisted of notification, history documentation, pre-pregnancy counselling, examinations, investigations, pharmacist referral, medications and surveillance. These indicators were selected from the CPG and Guidelines for the implementation and supervision of TB disease control programmes, TB disease control programme section, Sarawak state health department (June 2017 2nd edition)

Once indicators were chosen, a target level of performance was set as the consensus opinion among audit team members and a family medicine specialist. When a criterion is critical to the safety of service users, targets may be set at $100 \%$. For this reason, our level of target performance was set at $100 \%$.

This audit was carried out to ensure that PR1 KK Miri is managed according to the guidelines mentioned above. The required data obtained from manual files were recorded in Excel spreadsheets. Descriptive analysis was conducted using the Social Package of Social Sciences (SPSS) software and results are reported as frequencies and percentages. 


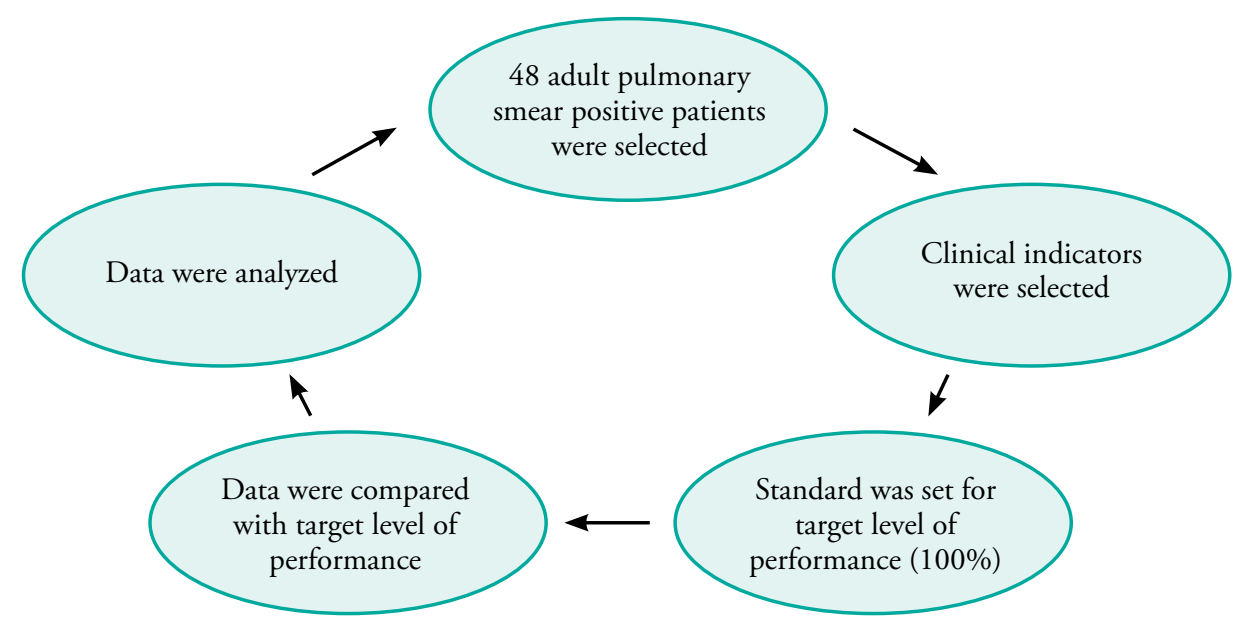

Figure 1. Conceptual framework for the audit regarding process of care

\section{Results}

There were 138 TB patients between August 2018 and August 2019. Only 48 of those patients were smear positive for PTB and were recruited into the audit. The sociodemographic profile of these patients is presented in Table 1 . The majority of patients were male (54.2\%) and Malay (33.2\%). Overall, $18.8 \%$ of patients were smokers and $4.2 \%$ were HIV positive.

Table 1. Sociodemographic profile of smear positive pulmonary tuberculosis patients $(n=48)$

\begin{tabular}{|c|c|}
\hline Variable & Number, Percentage (\%) \\
\hline $\begin{array}{l}\text { Gender } \\
\text { Female } \\
\text { Male }\end{array}$ & $\begin{array}{l}22(45.8) \\
26(54.2)\end{array}$ \\
\hline $\begin{array}{l}\text { Age groups } \\
<20 \\
20-29 \\
30-39 \\
40-49 \\
50-59 \\
60-69 \\
\geq 70\end{array}$ & $\begin{array}{c}2(4.2) \\
3(6.3) \\
11(22.9) \\
7(14.6) \\
10(20.8) \\
9(18.8) \\
6(12.5) \\
\end{array}$ \\
\hline $\begin{array}{l}\text { Ethnicity } \\
\text { Malay } \\
\text { Chinese } \\
\text { Iban } \\
\text { Other }\end{array}$ & $\begin{array}{c}16(33.3) \\
9(18.8) \\
12(25) \\
11(22.9) \\
\end{array}$ \\
\hline $\begin{array}{l}\text { Comorbidities } \\
\text { Hypertension } \\
\text { Diabetes } \\
\text { Dyslipidaemia } \\
\text { Heart disease } \\
\text { Other }\end{array}$ & $\begin{array}{c}9(18.8) \\
19(39.6) \\
4(8.3) \\
2(4.2) \\
16(33.3) \\
\end{array}$ \\
\hline $\begin{array}{l}\text { Smoking status } \\
\text { Smoker } \\
\text { Non-smoker } \\
\text { Ex-smoker }\end{array}$ & $\begin{array}{c}9(18.8) \\
27(56.3) \\
12(25) \\
\end{array}$ \\
\hline $\begin{array}{l}\text { HIV status } \\
\text { Positive } \\
\text { Negative }\end{array}$ & $\begin{array}{c}2(4.2) \\
46(95.8) \\
\end{array}$ \\
\hline $\begin{array}{l}\text { Drug status } \\
\text { Drug user } \\
\text { Non-drug user } \\
\text { Ex-drug user } \\
\text { Not recorded } \\
\end{array}$ & $\begin{array}{c}0(0) \\
42(87.5) \\
1(2.1) \\
5(10.4) \\
\end{array}$ \\
\hline $\begin{array}{l}\text { Alcohol status } \\
\text { Alcohol drinker } \\
\text { Non-alcohol drinker } \\
\text { Ex-drinker } \\
\text { Not recorded }\end{array}$ & $\begin{array}{c}1(2.1) \\
41(85.4) \\
0(0) \\
6(12.5) \\
\end{array}$ \\
\hline
\end{tabular}


Table 2. Indicators, criteria and target level of performance for audit of Pusat Rawatan 1 TB clinic at KK Bandar Miri

\begin{tabular}{|c|c|c|c|}
\hline No & Indicators & Criteria & Target level of performance (\%) \\
\hline 1. & Notification & - Case notified & 100 \\
\hline 2. & History documentation & $\begin{array}{l}\text { - TB symptoms assessed } \\
\text { - LMP } \text { LMPT }^{2} / \mathrm{UPT}^{2} \\
\text { - Medication side effects assessed }\end{array}$ & $\begin{array}{l}100 \\
100 \\
100\end{array}$ \\
\hline 3. & Pre-pregnancy counselling & - Case referred & 100 \\
\hline 4. & Examinations & $\begin{array}{l}\text { - Weight recorded } \\
\text { - Visual acuity } \\
\text { - Colour vision } \\
\text { - Respiratory rate recorded } \\
\text { - SP02 }{ }^{3} \text { recorded } \\
\text { - Lung findings }\end{array}$ & $\begin{array}{l}100 \\
100 \\
100 \\
100 \\
100 \\
100\end{array}$ \\
\hline 5. & Investigations & $\begin{array}{l}\text { - Baseline IX } \mathrm{IX}^{4 *} \\
\text { - CXR done and documented } \\
\text { - Sputum AFB } \mathrm{AF}^{5} 1, \mathrm{x} 2, \mathrm{x} 3 \\
\text { - Sputum MTB C\&S } \\
\text { - LFT X2/52 taken } \\
\text { - LFT X2/52 reviewed } \\
\text { - Other IX reviewed } \\
\text { - MTB C\&S traced }\end{array}$ & $\begin{array}{l}100 \\
100 \\
100 \\
100 \\
100 \\
100 \\
100 \\
100\end{array}$ \\
\hline 6. & Pharmacist referral & - Case referred & 100 \\
\hline 7. & Medications & - Anti-TB continued & 100 \\
\hline 8. & Surveillance & - Appointment x 6/12 given & 100 \\
\hline
\end{tabular}

${ }^{1}$ Last menstrual period, ${ }^{2}$ Urine pregnancy test, ${ }^{3}$ Oxygen saturation, ${ }^{4}$ Investigations, ${ }^{5}$ Sputum acid-fast bacilli, ${ }^{6}$ Mycobacterium tuberculosis culture and sensitivity, ${ }^{7}$ Liver function test

**Baseline investigations: renal profile, liver function test, full blood count, fasting blood sugar, HIV rapid test

Table 3. Process for measuring care of smear positive pulmonary tuberculosis patients

\begin{tabular}{|c|c|c|c|c|c|c|c|c|c|c|}
\hline \multirow{2}{*}{ No } & \multirow{2}{*}{ Indicators } & \multirow{2}{*}{ Criteria } & \multicolumn{8}{|c|}{ Results at month (\%) } \\
\hline & & & 0 & $2 / 52$ & 1 & 2 & 3 & 4 & 5 & 6 \\
\hline 1. & Notification & Case notified & 100 & & & & & & & \\
\hline \multirow[t]{3}{*}{2.} & \multirow{3}{*}{$\begin{array}{l}\text { History } \\
\text { documentation }\end{array}$} & Were TB symptoms assessed & 100 & 29.2 & 81.3 & 97.9 & 16.7 & 95.8 & 22.9 & 95.8 \\
\hline & & $\mathrm{LMP}^{1 / \mathrm{UPT}^{2}}$ & 4.2 & & 2.1 & 6.3 & 0 & 8.3 & 0 & 4.2 \\
\hline & & Medication side effects assessed & & 2.1 & 8.3 & 2.1 & 0 & 0 & 0 & 0 \\
\hline 3. & $\begin{array}{l}\text { Pre pregnancy } \\
\text { counselling }\end{array}$ & Case referred & 6.3 & & & & & & & \\
\hline \multirow[t]{8}{*}{4.} & \multirow[t]{5}{*}{ Examinations } & Weight recorded & 87.5 & 27.1 & 81.3 & 91.7 & 77.1 & 97.9 & 79.2 & 93.8 \\
\hline & & Visual acuity & 25 & & 0 & 0 & & & & \\
\hline & & Colour vision & 4.2 & & 0 & 0 & & & & \\
\hline & & Respiratory rate recorded & 41.7 & & 35.4 & 35.4 & & 29.2 & & 31.2 \\
\hline & & $\mathrm{SPO}^{3}{ }^{3}$ recorded & 45.8 & & 2.0 & 31.2 & & 10.4 & & 10.4 \\
\hline & \multirow[t]{3}{*}{ Lung findings } & Positive finding* & 23.0 & & 4.2 & 0 & & 0 & & 0 \\
\hline & & Normal finding & 70.8 & & 64.6 & 81.3 & & 85.4 & & 93.8 \\
\hline & & Not recorded & 6.2 & & 31.2 & 18.7 & & 14.6 & & 6.2 \\
\hline \multirow[t]{8}{*}{5.} & Investigations & Baseline IX $^{4 * *}$ & 100 & & & & & & & \\
\hline & & CXR done and documented & 100 & & & 87.5 & & 93.8 & & 95.8 \\
\hline & & Sputum $\mathrm{AFB}^{5} \mathrm{x} 1, \mathrm{x} 2, \mathrm{x} 3$ & 97.9 & 68.8 & 77.1 & 93.8 & 91.7 & 95.8 & 79.2 & 95.8 \\
\hline & & Sputum MTB C\&\& ${ }^{6}$ & 100 & & & & & & & \\
\hline & & $\mathrm{LFT}^{7} \mathrm{X} 2 / 52$ taken & & 83.3 & & & & & & \\
\hline & & LFT X2/52 reviewed & & & 83.3 & & & & & \\
\hline & & Other IX reviewed & & & 81.3 & & & & & \\
\hline & & MTB C\&S traced & & & & & & & & 81.3 \\
\hline 6. & $\begin{array}{l}\text { Pharmacist } \\
\text { referral }\end{array}$ & Case referred & 0 & & & & & & & \\
\hline 8. & Medications & Anti-TB continued & & & 100 & 100 & 100 & 100 & 100 & \\
\hline 9. & Surveillance & Appointment $\times$ 6/12 given & & & & & & & & 100 \\
\hline
\end{tabular}

${ }^{1}$ Last menstrual period, ${ }^{2}$ Urine pregnancy test, ${ }^{3}$ Oxygen saturation, ${ }^{4}$ Investigations, ${ }^{5}$ Sputum acidfast bacilli, ${ }^{6}$ Mycobacterium tuberculosis culture and sensitivity, ${ }^{7}$ Liver function test

** Baseline investigations: renal profile, liver function test, full blood count, fasting blood sugar, HIV rapid test;

* Reduced air entry, presence of crepitations 


\section{Discussion}

Tuberculosis remains an important public health disease and a challenge in Malaysia, despite the implementation of significant efforts in TB control interventions. ${ }^{7}$ Hence, appropriate and effective management with detailed documentation of PTB patients' data is important for giving the best care.

Regular monitoring of patients facilitates treatment completion, whereby monitoring response to treatment is achieved through regular history taking, physical examination, chest radiography (CXR) and laboratory monitoring. ${ }^{8,9}$ In our audit, we found that monitoring had been practiced as recommended but required further improvement. Some aspects of history were not taken and some examinations were not done. CXR was mostly done, but findings were not always documented.

All TB patients should be routinely assessed for comorbidities such as non-communicable diseases like diabetes, kidney or liver diseases, alcohol consumption, drug abuse and tobacco use. $^{7}$ A comorbidity history was taken and documented for all patients diagnosed. Efforts should be made to reduce modifiable risk factors to avoid worsening of TB. ${ }^{7}$ Every TB patient should be asked if they smoke and should be advised to quit with assistance. ${ }^{10}$ Smoking history is important and will be documented by clinic staff in TBIS 10A-1. Quitting smoking is important to reduce the risk of relapse after treatment completion and the risk of TB disease among the household contacts due to passive smoking. ${ }^{10}$

HIV testing was done for all patients at diagnosis and documented in patients' files. The definitive diagnosis of HIV infection rests on a positive ELISA test, so all confirmed TB patients must be offered HIV testing. ${ }^{10}$ One out of ten among the 10.4 million people that develop TB each year is HIV positive. ${ }^{11}$

Medical officers who diagnosed patients with TB are mandated to report the case to a nearby District Health Office in Malaysia, which will registered it in the MyTB system. ${ }^{7}$ The information provided through notification is used for epidemiological surveillance to control $\mathrm{TB}$ and to identify cases for cohort review. ${ }^{12}$ All smear positive PTB patients in our clinic were notified at diagnosis, as per the guideline.

It is evident from the audit that TB symptoms were assessed more frequently at follow-up months one, two, four and six, as they were seen by medical officers at those times. At the second week, and third and fifth month follow up, however, only $29.2 \%, 16.7 \%$ and $22.9 \%$ of patients, respectively, had their symptoms assessed, as they were seen only by our clinic staff at those visits. These symptom assessments are important because they ensure that patients are clinically improving after initiation of treatment.

Medication side effects were assessed minimally for two months of anti-TB treatment and then not assessed at all for subsequent follow ups. This is likely due to either patients not complaining during the anti-TB treatment or this assessment being taken lightly because most patients complete their treatment without any significant adverse drug effects. It is important that patients are clinically monitored during treatment with good documentation so that adverse effects can be detected promptly and managed properly. ${ }^{7,8}$ Health personnel can monitor adverse drug effects by teaching patients how to recognise the symptoms of common side effects. ${ }^{8}$

According to the National Strategic Plan for Tuberculosis Control (2016 - 2020), the majority of $\mathrm{TB}$ cases reported are from the reproductive age group; in 2015, 53\% of TB cases occurred among people 25 - 54 years of age. ${ }^{7}$ This further justifies the importance of PPC and LMP/UPT history at each TB follow up. Of the 48 patients in this audit, 22 were female but only 14 were of reproductive age. Of these, only three $(6.3 \%)$ were referred for PPC. LMP/UPT history was not taken consistently during each follow up. Women of childbearing age should be asked about current or planned pregnancy prior to starting anti-TB drugs because anti-TB medications can lead to perinatal complications. Maternal TB has been associated with increased risk of maternal mortality and perinatal morbidity, namely premature delivery, small-for-gestation age and low birth weight. ${ }^{1}$

Patients' weights should be monitored and documented monthly, and dosages should be adjusted if weight changes to prevent underor overdosing. ${ }^{8}$ Weight monitoring is therefore a salient piece of data to record during each follow up.

Baseline vision tests for visual acuity and colour perception (e.g. using the Snellen and Ishihara 
charts) should be conducted before starting treatment. ${ }^{13}$ Our audit demonstrated, however, that visual acuity and colour vision had only been tested in $12(25 \%)$ and two (4.2\%) patients, respectively, at first diagnosis. No further repeated examinations were conducted at one and two month follow ups, either. Worsening from the baseline vision which is due to Ethambutol may occasionally cause ocular toxicity, but evidence suggests that it is safe provided proper precautions are taken when prescribing the drug. ${ }^{13}$

It is essential to highlight that baseline investigations were taken for all 48 patients at diagnosis. Only 39 (81.3\%) patients' blood results were traced and reviewed at the onemonth follow up, however. This can lead to missed abnormal results or unnecessary continuation of anti-TB treatment. Patients with liver and renal impairment may require frequent monitoring while on treatment, as they may develop side effects due to treatment or may end up receiving inadequate therapy. ${ }^{1}$

Radiographic images are some of the most important investigations used, particularly in diagnosing PTB. ${ }^{14}$ In our audit, we found that most of the TB patients had a CXR done and $100 \%, 87.5 \%, 93.8 \%$ and $95.8 \%$ of patients had their CXR reviewed and recorded at diagnosis and after two months, four months and six months, respectively. The target level of performance for CXR was $100 \%$, as CXR review and documentation help to monitor patients' progression and to decide on continuation of anti-TB treatment. ${ }^{14}$

Sputum specimens should be collected for smear examination at each monthly follow up, as it helps to predict treatment outcomes. ${ }^{8,14}$ Based on the above audit, sputum smears were frequently missed in some patients or were done but results were not reviewed and documented. This could be explained by patients defaulting their appointments to send sputum for acid-fast bacilli testing due to transport problems or staying further from the clinic.

Sputum culture is used not only as a confirmatory method in detecting the presence of $M$. tuberculosis, but also for screening of primary drug susceptibility and resistance. ${ }^{14}$ Sputum culture is necessary to monitor the progress of treatment as it is more sensitive. ${ }^{9} \mathrm{~A}$ total of $81.3 \%$ of $M$. tuberculosis culture and sensitivity has been traced by the time patient is discharged. It is important to trace all culture and sensitivity results, especially if neither the CXR nor the patient is improving clinically, despite undergoing anti-TB treatment.

One of the most common side effects of antiTB treatment is liver injury, which can be detected earlier with proper liver function monitoring. ${ }^{15}$

Medication counselling by pharmacists, especially upon initiation of treatment, could enhance patients' adherence by increasing patients' understanding about the importance of compliance and the side effects of treatment. ${ }^{7}$ In our audit, no patients were referred to a pharmacist. Brief counselling was done by our staff upon initiation of anti-TB treatment.

All patients were given six months of surveillance follow up, as per the guideline. ${ }^{16}$ This follow up is important to watch for symptoms of relapse. $^{2}$ All 48 patients completed their anti-TB medications. Two patients, however, completed their anti-TB treatment over a period of seven months, with the intensive phase extended to three months in view of persistent positive smears at two months post-diagnosis. No treatment failure or defaulters were found throughout the audit.

Overall, there were some downfalls in certain aspects and documentation. This is most likely due to patient overload, a lack of knowledge concerning the importance of some documentation and manpower constraints. Our committed staff members must handle blood taking, Mantoux test injection and reading, contact tracing, MyTB system updates, appointments and all administrative work.

\section{Conclusion}

This audit shows there is a difference between clinical practice and the guidelines when it comes to providing ideal care for patients through comprehensive history and examination with proper documentation.

This audit was, however, subject to several limitations. Firstly, our sample size collection was not based on target population, as it was a retrospective data collection over a one-year time frame. The reason for this was that data collection for the audit occurred in March 2020, which was six months after all patients completed anti-TB treatment. The second limitation was the incompletion of the audit cycle. Implementations must be carried out 
and a re-audit should be done after six months in order to look for improvements following action plans.

Recommendations include placing a comprehensive checklist in each patient's file during registration, training staff members to multitask and conducting continuing medical education to update staff members on current CPG.

\section{Funding}

None.

\section{Conflicts of Interest}

All authors declare no conflicts of interest.

\section{Ethics Approval}

The audit was registered under the National Medical Research Registry with registration number NMRR-20-1893-56000. All medical records data were obtained from PR1, KK Bandar Miri and patients' information will be kept confidential.

\section{How does this paper make a difference to general practice?}

- Determines pitfalls for primary care doctors regarding clinical documentation for PTB patients

- Creates awareness among healthcare workers concerning the importance of proper documentation for overall management

- Provides evidence to improve clear and accurate clinical documentation

\section{References}

1. World Health Organization. Global tuberculosis report 2014. Geneva: World Health Organization; 2014. Available from: http://apps.who.int/iris/handle/ 10665/137094

2. Ministry of Health Malaysia. Clinical Practice Guidelines for Management of Tuberculosis Third edition. Putrajaya: MaHTAS. 2012: 1-49

3. Escreet BC, Cowie RL. Criteria for the diagnosis of pulmonary tuberculosis. S Afr Med J. 1983;63(22): 850-854

4. Limb C, Fowler A, Gundogan B, Koshy K, Agha R. How to conduct a clinical audit and quality improvement project. Int J Surg Oncol (N Y). 2017;2(6):e24. doi:10.1097/ IJ9.0000000000000024

5. Mathioudakis A, Rousalova I, Gagnat AA, Saad N, Hardavella G. How to keep good clinical records. Breathe (Sheff). 2016;12(4): 369-373. doi: $10.1183 / 20734735.018016$

6. Williams G, Alarcon E, Jittimanee S, Walusimbi M, Sebek M, Berga E, Villa TS. Guidance for the implementation of best practice for the care of patients with tuberculosis. Int J Tuberc Lung Dis. 2008;12(3): 19 .
7. Disease Control Division (TB/ Leprosy Sector) Ministry of Health Malaysia.National strategic plan for tuberculosis control (2016 - 2020). Putrajaya: Ministry of Health Malaysia; 2016: $1-28$

8. World Health Organization. Treatment of tuberculosis: guidelines for national programmes (fourth edition). Geneva: World Health Organization; 2010. Available from: https:/www.who.int/tb/ publications/2010/9789241547833/en/

9. World Health Organization. Companion handbook to the WHO guidelines for the programmatic management of drugresistant tuberculosis. Geneva: World Health Organization; 2014. Monitoring Treatment Response. Available from: https://www.who.int/ tb/publications/pmdt_companionhandbook/en/

10. National Department of Health South Africa. National Tuberculosis Management Guideline. South Africa: Directorate of TB DOTS Strategy Coordination. 2014: 11-70
11. Ramírez S, Mejía F, Rojas M, Seas C, Stuyft PVD, Gotuzzo E, Otero L. HIV screening among newly diagnosed TB patients: A cross sectional study in Lima, Peru. BMC Infect Dis. 2018;18(1): 136. Published 2018 Mar 20.doi:10.1186/s12879-018-3037-5

12. Surinder Tamne. Guidance on notifying Tuberculosis (TB) cases. England PHE publications gateway number: 2014370.2014;1. Available from: https://assets. publishing.service.gov.uk/government/uploads/ system/uploads/attachment_data/file/360263/ Guidance_on_Notifying_Tuberculosis_TB_ cases.pdf

13. Ming TC, Wai YW, Chiu LC, Choi CY. Annual Report 2002, Tuberculosis \& Chest Service of The Department Of Health. Hong Kong; TBCCC/TBSC Department of Health and the Hospital Authority; 2014. Preventive measures against drug-induced ocular toxicity during anti-tuberculosis treatment: Available from: https://www.info.gov.hk/tb_chest/doc/ Annurept_2002.pdf 
14. Nissapatorn V, Lim YAL, Jamaiah I, Chin HM, Ilyana MZ, Nonaziah MZ, Hasifah AS, Kuppusamy I. Tuberculosis in Malaysia: A continuing surge. Southeast Asian J Trop Med Public Health. 2007; 38: 236-237
15. Chang TE, Huang YS, Su WJ, Perng CL, Huang YH, Hou MC. The role of regular liver function monitoring in antituberculosis drug-induced liver injury. J Chin Med Assoc. 2019;82(7): 535-540. doi:10.1097/ JCMA.0000000000000119
16. Sarawak State Health Department. TB disease control programme section, Sarawak state health department Guidelines for the implementation and supervision of TB disease control Programmes Second edition. Sarawak: Sarawak state health department. 2017. 